\title{
Title: INFERRING DIFFERENTIAL LEUKOCYTE ACTIVITY FROM ANTIBODY MICROARRAYS USING A LATENT VARIABLE MODEL
}

DOI No: $10.1142 / 97818481633240011$

Source: GENOME INFORMATICS 2008 Genome Informatics Series Vol. 21 (pp 126-137)

Author(s): JOSHUA W.K. HO

School of Information Technologies, The University of Sydney, NSW 2006, Australia NICTA, Australia

RAJEEV KOUNDINYA

Bosch Institute, The University of Sydney, NSW 2006, Australia

TIBÉRIO S. CAETANO

RSISE, Australian National University, ACT 2601, Australia

NICTA, Australia

CRISTOBAL G. DOS REMEDIOS

Bosch Institute, The University of Sydney, NSW 2006, Australia

MICHAEL A. CHARLESTON

School of Information Technologies, The University of Sydney, NSW 2006, Australia

Sydney Bioinformatics, The University of Sydney, NSW 2006, Australia

Centre for Mathematical Biology, The University of Sydney, NSW 2006, Australia

Abstract: Recent development of cluster of differentiation (CD) antibody arrays has enabled expression levels of many leukocyte surface CD antigens to be monitored simultaneously. Such membrane-proteome surveys have provided a powerful means to detect changes in leukocyte activity in various human diseases, such as cancer and cardiovascular diseases. The challenge is to devise a computational method to infer differential leukocyte activity among multiple biological states based on antigen expression profiles. Standard DNA microarray analysis methods cannot accurately infer differential leukocyte activity because they often fail to take the cell-to-antigen relationships into account. Here we present a novel latent variable model (LVM) approach to tackle this problem. The idea is to model each cell type as a latent variable, and represent the class-to-cell and cell-to-antigen relationships as a LVM. Once the parameters of the LVM are learned from the data, differentially active leukocytes can be easily identified from the model. We describe the model formulation and assumptions which lead to an efficient expectation-maximization algorithm. Our LVM method was applied to re-analyze two cardiovascular disease datasets. We show that our results match existing biological knowledge better than other methods such as gene set enrichment analysis. Furthermore, we discuss how our approach can be extended to become a general framework for gene set analysis for DNA microarrays.

Keywords: antibody microarray; latent variable model; Bayesian network; EM algorithm

Full Text: View full text in PDF format (1001KB)

TOC: Back to Table of Contents 